\title{
Caracterización de los pacientes con endocarditis infecciosa en población de Boyacá Estudio transversal
}

\author{
Characteristics of infectious endocarditis patients \\ from a town in Boyacá \\ A cross-sectional study
}

\author{
Javier Orlando Barón-Barón, Edwar Jassir Rozo-Ortiz, María Mónica Martínez-Mariño, \\ Fransol Najar-Molano, Ledmar Jovanny Vargas-Rodríguez, \\ Benjamín Alexander Márquez-Rosales • Tunja (Colombia)
}

DOI: https://doi.org/10.36104/amc.2020.1720

\section{Resumen}

La endocarditis infecciosa (EI) es una enfermedad multisistémica que resulta de una infección, generalmente bacteriana, de la superficie endocárdica. Su incidencia es de tres a nueve casos por cada 100000 personas.

Objetivo: realizar una caracterización de los pacientes atendidos en el Hospital Universitario San Rafael de Tunja con diagnóstico de endocarditis infecciosa.

Material y métodos: estudio observacional descriptivo. Pacientes atendidos en el Hospital Universitario San Rafael de Tunja entre el $1^{\circ}$. de enero del 2010 a $1^{\circ}$. de enero del 2019. Se tomaron variables sociodemográficas, clínicas, paraclínicas, imagenológicos, terapéuticas y de desenlaces.

Resultados: en total se atendieron 87 personas que presentaron diagnóstico confirmado de endocarditis infecciosa. La edad promedio fue 38.77 años con una DE \pm 13.32 años. Se observó leucocitosis en $90 \%$, neutrofilia en $85 \%$, linfocitosis en $3 \%$, trombocitopenia en $4 \%$, aumento de los valores séricos de PCR en $85 \%$, transaminitis en $45 \%$, hiponatremia en $2 \%$ e hipernatremia en $4 \%$. Todos los pacientes recibieron manejo antibiótico, tan solo $23 \%$ requirieron manejo quirúrgico.

Conclusiones: en esta serie de casos se encontraron características clínicas y microbiológicas similares a lo reportado en otras investigaciones nacionales e internacionales. Afectando con mayor frecuencia a personas del sexo masculino, siendo el principal agente causal el Staphylococcus aureus, el agente causal. La fiebre y los signos de vasculitis infecciosa fueron las principales manifestaciones clínicas, afectado con mayor frecuencia la válvula mitral. (Acta Med Colomb 2020; 45. DOI: https://doi.org/10.36104/amc.2020.1720).

Palabras clave: endocardio, endocarditis, válvulas cardiacas, bacteriemia, diagnóstico por imagen (DeCS).

\begin{abstract}
Infectious endocarditis (IE) is a multisystemic disease caused by an infection, generally bacterial, of the endocardial surface. Its incidence is three to nine cases per 100,000 persons.

Objective: to characterize the patients with a diagnosis of infectious endocarditis seen at the Hospital Universitario San Rafael de Tunja.

Materials and methods: a descriptive observational study. Patients seen at the Hospital Universitario San Rafael de Tunja between January 1, 2010 and January 1, 2019. Sociodemographic, clinical, paraclinical, imaging, treatment and outcome variables were gathered.

Results: a total of 87 persons with a confirmed diagnosis of infectious endocarditis were seen. The average age was 38.77 years, with an SD \pm 13.32 years. Leukocytosis was seen in $90 \%$, neutrophilia in $85 \%$, lymphocytosis in $3 \%$, thrombocytopenia in $4 \%$, elevated serum CRP in $85 \%$, transaminitis
\end{abstract}

Drs. Javier Orlando Barón, Edwar Jassir RozoOrtiz, María Mónica Martínez-Mariño, Fransol Najar-Molano: Departamento de Medicina Interna Hospital San Rafael, Facultad de Ciencias de la Salud Universidad de Boyacá; Dr. Fransol Najar-Molano: Facultad de Ciencias de la Salud Universidad de Boyacá; Dr. Ledmar Jovanny Vargas-Rodríguez: Departamentos de Medicina Interna y de Neurología, Hospital San Rafael; Dr. Benjamín Alexander Márquez-Rosales: Departamento de Neurología Hospital San Rafael. Tunja (Colombia).

Correspondencia: Dr. Ledmar Jovanny VargasRodríguez. Tunja (Colombia).

E-mail: lejovaro@gmail.com

Recibido: 02/III/2020 Aceptado: 22/VII/2020 
in $45 \%$, hyponatremia in $2 \%$ and hypernatremia in $4 \%$. All patients received antibiotic treatment; only $23 \%$ required surgery.

Conclusions: this case series found similar clinical and microbiological characteristics to those reported in other national and international studies. Males were affected more often than females, with the main etiological agent being Staphylococcus aureus. Fever and infectious vasculitis signs were the main clinical manifestations, most often affecting the mitral valve. (Acta Med Colomb 2020; 45. DOI: https://doi.org/10.36104/amc.2020.1720).

Key words: endocardium, endocarditis, heart valves, bacteremia, diagnostic imaging (DeCS).

\section{Introducción}

La endocarditis infecciosa (EI) es una enfermedad multisistémica que resulta de una infección, generalmente bacteriana de la superficie endocárdica del corazón. En la actualidad esta entidad patológica se clasifica de acuerdo con la situación diagnóstica (definitiva o posible), al sitio anatómico (izquierda/derecha), el tipo de válvula afectada (válvula nativa o protésica) y al microorganismo implicado (bacteria, hongo) (1). Su incidencia es de tres a nueve casos por cada 100000 personas con factores de riesgo $(2,3)$.

Dentro de la fisiopatología se ha logrado comprender que para el desarrollo de esta enfermedad se requiere la aparición simultánea de varios factores independientes: la alteración de la superficie de la válvula cardiaca para producir un sitio adecuado para la inserción y colonización bacteriana; bacteriemia con un organismo capaz de adherirse y colonizar tejido valvular; y la creación de la masa o "vegetación" infectada al "enterrar" el organismo en proliferación dentro de una matriz protectora de moléculas de suero por ejemplo, fibrina y plaquetas. La EI rara vez resulta de inyecciones intravenosas de bacterias a menos que la superficie valvular se perturbe por primera vez. En los seres humanos un daño equivalente a la superficie valvular puede deberse a una variedad de factores, incluido el flujo sanguíneo turbulento relacionado con el daño valvular primario, debido a estados de enfermedad sistémica específica como la carditis reumática, lesiones mecánicas causadas por catéteres o electrodos. Este daño endotelial provoca la formación de depósitos de fibrina-plaquetas sobre el edema intersticial, una entidad fisiopatológica denominada por primera vez "endocarditis trombótica no bacteriana" (3).

Esta genera cambios patológicos característicos en múltiples órganos diana. Las porciones de la matriz de plaquetas y fibrina de la vegetación pueden desprenderse de la válvula cardiaca infectada y viajar con sangre arterial hasta que se alojan en un lecho vascular. Tales émbolos sépticos pueden involucrar a casi cualquier sistema de órganos en el cuerpo y pueden manifestarse clínicamente de varias maneras. Este es el proceso patogénico de los accidentes cerebrovasculares embólicos, infarto de miocardio, riñón, bazo, mesenterio y piel $(3,4)$.

El objetivo del siguiente manuscrito es realizar una caracterización de los pacientes atendidos en el Hospital Universitario San Rafael de Tunja con diagnóstico de EI.

\section{Material y métodos}

Se realizó un estudio observacional, descriptivo, de corte transversal y retrospectivo. El universo del estudio fueron los pacientes atendidos en el Hospital Universitario San Rafael de Tunja entre el $1^{\circ}$. de enero del 2010 al $1^{\circ}$. de enero de 2019 con diagnóstico de EI, donde se incluyeron los relacionados con los códigos CIE-10 de la patología (I33.0, I33.9, I38.0, I39.0), mayores de 18 años de edad y se excluyeron pacientes con datos incompletos, remitidos para manejo en otra institución o que no cumplieran los criterios de inclusión ya mencionados.

En la historia clínica se revisaron las siguientes variables: sociodemográficas (edad, sexo y comorbilidades), clínicas (signos y síntomas), paraclínicas (hemograma, reactantes de fase aguda, electrolitos, función hepática y renal), microbiológicas (hemocultivos), imágenes (ecocardiograma, FEVI y medidas de las vegetaciones), terapéuticas (manejo antibiótico y quirúrgico) y de desenlaces (complicaciones y secuelas).

El análisis univariado se realizó por medio de un estadístico descriptivo a la población seleccionada, determinando frecuencias absolutas y relativas en las variables categóricas (con los respectivos intervalos de confianza a 95\% (IC 95\%), en el caso de las variables cuantitativas se calcularon las medidas de tendencia central (media, mediana) y medidas de dispersión (desviación estándar y rango intercuartil). La base de datos se registró en Excel versión 2013 y se analizó en el paquete estadístico SPSS versión 22, (C) Copyrigth IBM corporation (Licencia IBM Z125-3301-14). Basados en la resolución 8430 de 1993, se considera una investigación sin riesgo, para la revisión de las historias clínicas se obtuvo el debido permiso por parte del comité de ética e investigación del Hospital Universitario San Rafael de Tunja.

\section{Resultados}

\section{Características demográficas de la población}

En total se atendieron 87 personas que presentaron diagnóstico confirmado de endocarditis infecciosa. El promedio de edad fue 38.77 años con una DE \pm 13.32 años, el $72 \%$ eran del sexo masculino, residentes en zona rural y con ciertas comorbilidades que se muestran en la Tabla 1.

\section{Características clínicas y paraclínicas}

El tiempo de evolución del cuadro clínico varió entre uno a 30 días, con un promedio de 9.28 días ( $\mathrm{DE} \pm 7.56$ 
Tabla 1. Prevalencia por sexo, zona de residencia y comorbilidades presentes en los pacientes con diagnóstico de endocarditis infecciosa.

\begin{tabular}{|c|c|c|c|c|}
\hline Variable & $\mathbf{n}$ & $\%$ & \multicolumn{2}{|c|}{ IC $95 \%$} \\
\hline Sexo & & & & \\
\hline Femenino & 24 & 27.6 & 18.2 & 36.98 \\
\hline Masculino & 63 & 7.4 & 63.02 & 81.8 \\
\hline \multicolumn{5}{|l|}{ Residencia } \\
\hline Rural & 55 & 63.2 & 53.09 & 73.35 \\
\hline Urbana & 32 & 36.8 & 26.65 & 46.91 \\
\hline \multicolumn{5}{|l|}{ Comorbilidades } \\
\hline Diabetes mellitus & 28 & 3.2 & 22.37 & 42,0 \\
\hline HTA & 47 & 54.0 & 43.55 & 64.49 \\
\hline EPOC & 16 & 18.4 & 10.25 & 26.53 \\
\hline SPA & 8 & 9.2 & 3124.0 & 15.27 \\
\hline Cardiopatía congénita & 8 & 9.2 & 3124.0 & 15.27 \\
\hline Valvulopatías & 34 & 39.1 & 28.83 & 49.33 \\
\hline IVU último mes & 21 & 24.1 & 15.15 & 33.13 \\
\hline Cirugía cardiaca & 4 & 4.6 & 0.19 & 8.98 \\
\hline Implantes dentales & 21 & 24.1 & 15.15 & 33.13 \\
\hline Enfermedad autoinmune & 22 & 25.3 & 16.15 & 34.42 \\
\hline Enfermedad mental & 11 & 12.6 & 5.66 & 19.63 \\
\hline Enfermedad renal (aguda/crónica) & 21 & 24.1 & 15.15 & 33.13 \\
\hline Arritmias & 32,0 & 36.8 & 26.65 & 46.91 \\
\hline Faringoamigdalitis & 40.0 & 46.0 & 35.51 & 56.45 \\
\hline
\end{tabular}

días), siendo la fiebre y los signos de vasculitis infecciosa sistemática las manifestaciones más frecuentes (Tabla 2).

Los microorganismos gram positivos fueron los que se encontraron con mayor frecuencia en los hemocultivos (Tabla 2). Se observó leucocitosis en $90 \%$, neutrofilia en $85 \%$, linfocitosis en $3 \%$, trombocitopenia en $4 \%$, aumento de los valores séricos de PCR en $85 \%$, transaminitis en $45 \%$, hiponatremia en $2 \%$ e hipernatremia en el $4 \%$ de los casos (Tabla 2).

\section{Características imagenológicos}

Para el diagnóstico mediante el ecocardiograma transtorácico (ETT) se diagnosticaron la mayor parte de los pacientes $(94.3 \%)$, tan solo $5.7 \%$ requirieron ecocardiograma transesofágico (ETE) para la confirmación del diagnóstico. Las válvulas miocárdicas más afectadas fueron la mitral y la aórtica, en donde la mayoría presentaban regurgitación moderada y tan sólo $4.6 \%$ eran válvulas protésicas (Tabla 3).

El promedio de fracción de eyección ventricular izquierda (FEVI) fue $60.67 \%$ (DE $\pm 16.85 \%$ ). Las vegetaciones tenían
Tabla 2. Características clínicas y paraclínicas en pacientes diagnosticados con endocarditis infecciosa. Microorganismos etiopatogénicos aislados.

\begin{tabular}{|c|c|c|c|c|}
\hline Manifestaciones clínicas & $\mathbf{n}$ & $\%$ & \multicolumn{2}{|c|}{ IC95\% } \\
\hline Fiebre & 73.0 & 83.9 & 76.19 & 91.63 \\
\hline Vasculitis infecciosa & 53.0 & 60.9 & 50.67 & 71.17 \\
\hline Nódulos de Osler & 12.0 & 13.8 & 5.66 & 19.63 \\
\hline Taquicardia & 20.0 & 23.0 & 14.15 & 31.83 \\
\hline Astenia/adinamia & 70.0 & 80.5 & 72.13 & 88.79 \\
\hline Soplo cardiaco & 69 & 79.3 & 70.8 & 87.82 \\
\hline Esplenomegalia & 36 & 41.4 & 31.83 & 51.33 \\
\hline Tos & 12 & 13.8 & 5.66 & 19.63 \\
\hline Disnea & 28 & 32.2 & 22.37 & 42 \\
\hline Dolor torácico & 24 & 27.6 & 18.2 & 36.98 \\
\hline Accidente cerebrovascular & 13 & 14.9 & 7.45 & 22.43 \\
\hline Cambios de comportamiento & 4 & 4.6 & 0.19 & 8.98 \\
\hline Náuseas y vómitos & 8 & 9.2 & 3.12 & 15.27 \\
\hline \multicolumn{5}{|c|}{ Microorganismos causantes } \\
\hline S. Aureus & 31 & 35.6321839 & 26.65 & 46.91 \\
\hline Streptococcus & 28 & 32.183908 & 22.37 & 42 \\
\hline Klebsiella pneumoniae & 8 & 9.954023 & 3.12 & 15.27 \\
\hline S.epidermidis & 7 & 8.04597701 & 2.31 & 13.76 \\
\hline Enterobacter & 3 & 3.44827586 & 0.0 & 7.282 \\
\hline Cultivos negativos & 10 & 11.4942529 & 4.79 & 18.2 \\
\hline \multicolumn{5}{|c|}{ Características de los paraclínicos } \\
\hline Paraclínico & \multicolumn{2}{|c|}{ Promedio } & \multicolumn{2}{|c|}{ Desviación estándar } \\
\hline Leucocitos $\left(\mathrm{mm}^{3}\right)$ & \multicolumn{2}{|c|}{16806.78} & \multicolumn{2}{|c|}{4291.635} \\
\hline Plaquetas $\left(\mathrm{mm}^{3}\right)$ & \multicolumn{2}{|c|}{264942.53} & \multicolumn{2}{|c|}{106045.663} \\
\hline Proteína C reactiva $(\mathrm{mg} / \mathrm{dL})$ & \multicolumn{2}{|c|}{31.9802} & \multicolumn{2}{|c|}{30.23511} \\
\hline Neutrófilos (\%) & \multicolumn{2}{|c|}{82.826} & \multicolumn{2}{|c|}{7.3225} \\
\hline Linfocitos $(\%)$ & \multicolumn{2}{|c|}{9.262} & \multicolumn{2}{|c|}{6.4518} \\
\hline Bilirrubina directa $(\mathrm{mg} / \mathrm{dL})$ & \multicolumn{2}{|c|}{0.9425} & \multicolumn{2}{|c|}{0.51194} \\
\hline Bilirrubina indirecta $(\mathrm{mg} / \mathrm{dL})$ & \multicolumn{2}{|c|}{0.3143} & \multicolumn{2}{|c|}{0.17552} \\
\hline AST / GOT (U/L) & \multicolumn{2}{|c|}{85.24} & \multicolumn{2}{|c|}{106.529} \\
\hline ALT / GPT (U/L) & \multicolumn{2}{|c|}{75.86} & \multicolumn{2}{|c|}{102.558} \\
\hline Creatinina $(\mathrm{mg} / \mathrm{dL})$ & \multicolumn{2}{|c|}{1.7501} & \multicolumn{2}{|c|}{1.13135} \\
\hline Sodio $(\mathrm{mEq} / \mathrm{L})$ & \multicolumn{2}{|c|}{143.051} & \multicolumn{2}{|c|}{9.5146} \\
\hline Potasio $(\mathrm{mEq} / \mathrm{L})$ & \multicolumn{2}{|c|}{3.9690} & \multicolumn{2}{|c|}{0,46554} \\
\hline
\end{tabular}

un promedio de ancho de $1.10 \mathrm{~cm}(\mathrm{DE} \pm 0.49 \mathrm{~cm})$ y con una altitud promedio de $0.85 \mathrm{~cm}(\mathrm{DE} \pm 0.23 \mathrm{~cm})$.

\section{Tratamiento, complicaciones y secuelas}

Todos los pacientes recibieron manejo antibiótico por seis semanas siguiendo el protocolo institucional y sólo el $23 \%$ 
Tabla 3. Características imagenológicas, con evidencia de afección en las válvulas miocárdicas, donde la mayoría presentaban regurgitación moderada y solo el $4.6 \%$ eran válvulas protésicas.

\begin{tabular}{|c|c|c|c|c|}
\hline & $\mathbf{n}$ & $\%$ & \multicolumn{2}{|c|}{ IC $95 \%$} \\
\hline \multicolumn{5}{|c|}{ Ecocardiograma } \\
\hline ETE & 5.0 & 5.7 & 0.8568 & 10.64 \\
\hline ETT & 82.0 & 94.3 & 89.36 & 99.14 \\
\hline \multicolumn{5}{|c|}{ Válvula afectada } \\
\hline Aórtica & 16.0 & 18.4 & 89.36 & 99.14 \\
\hline Mitral & 59.0 & 67.8 & 57.09 & 77.35 \\
\hline Mitral y aórtica & 8.0 & 9.2 & 3.12 & 15.27 \\
\hline Tricúspide & 4.0 & 4.6 & 0.19 & 8.98 \\
\hline \multicolumn{5}{|c|}{ Grado de regurgitación } \\
\hline Leve & 24.0 & 27.6 & 18.2 & 36.98 \\
\hline Moderada & 50.0 & 57.5 & 47.08 & 67.86 \\
\hline Severa & 13.0 & 14.9 & 7.45 & 22.43 \\
\hline \multicolumn{5}{|l|}{ Válvula nativa } \\
\hline No & 4.0 & 4.6 & 0.19 & 8.98 \\
\hline Sí & 83.0 & 95.4 & 76.19 & 91.63 \\
\hline
\end{tabular}

requirieron manejo quirúrgico, entre ellos tres que finalmente requirieron inserción de marcapasos por la gravedad de la patología (presentaron bloqueo AV de alto grado).

Se observó que las complicaciones que se presentaron con mayor frecuencia fueron las arritmias y la falla cardiaca, siendo esta última una secuela importante para muchos pacientes (Tabla 4).

\section{Discusión}

La EI es una enfermedad infecciosa sistémica potencialmente mortal (4). A pesar de que la EI es una patología rara, su incidencia ha aumentado en los países desarrollados y hay pocos estudios disponibles en los países en desarrollo $(4,5)$. La endocarditis es el cuarto síndrome infeccioso más importante después de la urosepsis, la neumonía y la sepsis intraabdominal en los departamentos médicos de los países desarrollados (6).

Se observó que en la población estudiada existe una mayor prevalencia de endocarditis infecciosa en el género masculino con $72.4 \%$ similar a los reportes en los estudios latinoamericanos y a nivel mundial $(7,8)$. En cuanto a la edad el promedio fue de 38.77 años, con una $\mathrm{DE} \pm 13.32$ años en su mayoría residentes de las zonas rurales. En esta investigación los principales agentes etiológicos identificados en hemocultivo fueron S. Aureus (35.6\%), Streptococcus (32.1\%), Klebsiella pneumoniae (9\%). Disociando con algunos estudios donde se reportó incidencia de agentes como Enterococcus spp al igual que una prevalencia de los ya mencionados (9).

La fiebre fue la principal manifestación clínica seguida por astenia, adinamia, encontrado de igual forma una alta
Tabla 4. Complicaciones más frecuentes y sus posteriores secuelas encontradas en los pacientes diagnosticados con endocarditis infecciosa en el departamento de Boyacá.

\begin{tabular}{|l|c|c|c|c|}
\hline \multicolumn{1}{|c|}{ Complicaciones } & $\mathbf{n}$ & $\%$ & \multicolumn{2}{c|}{ IC 95\% } \\
\hline Embolia pulmonar & 12.0 & 13.8 & 5.66 & 19.63 \\
\hline Embolia cerebral & 12.0 & 13.8 & 5.66 & 19.63 \\
\hline Pericarditis & 16.0 & 18.4 & 10.25 & 26.53 \\
\hline Miocarditis & 5.0 & 5.7 & 0.8568 & 10.64 \\
\hline Falla cardiaca & 31.0 & 35.6 & 26.65 & 46.91 \\
\hline Enfermedad renal & 5.0 & 5.7 & 0.8568 & 10.64 \\
\hline Arritmias & 24.0 & 27.6 & 17.35 & 36.82 \\
\hline Absceso cerebral & 14.0 & 16.1 & 8.45 & 24.43 \\
\hline Embolia coronaria & 8.0 & 9.2 & 3124.0 & 15.27 \\
\hline Derrame pleural & 12,0 & 13,8 & 7.45 & 22.43 \\
\hline Derrame pericárdico & 7.0 & 8.0 & 2.31 & 13.76 \\
\hline Secuelas (n=44) & 4.0 & 4.6 & 0.19 & 8.98 \\
\hline Hemiparesia y falla cardiaca & 7.0 & 8.0 & 2.31 & 13.76 \\
\hline Hipertensión pulmonar & 8.0 & 9.2 & 3.12 & 15.27 \\
\hline Falla cardiaca & 15.0 & 17.2 & 9304,0 & 25.18 \\
\hline Arritmias e falla cardiaca & 5.0 & 5.7 & 0.8568 & 10.64 \\
\hline $\begin{array}{l}\text { Arritmias, enfermedad } \\
\text { renal y falla cardiaca }\end{array}$ & 5.0 & 5.7 & 0.8568 & 10.64 \\
\hline Paresia de una extremidad & & & & \\
\hline
\end{tabular}

prevalencia de soplo cardiaco y vasculitis infecciosa, coincidiendo con el cuadro reportado en otras investigaciones (10-12). Dentro de los síntomas menos frecuentes están: tos, nódulos de Osler y cambios en el comportamiento. Las válvulas miocárdicas más afectadas fueron la mitral y la aórtica, en donde la mayoría presentaban regurgitación moderada y tan solo $4.6 \%$ eran válvulas protésicas, reporte similar por otros autores $(13,14)$. La precisión diagnóstica de la ecocardiografía transtorácica para detectar signos ecocardiográficos de endocarditis infecciosa es alta; pero en aquellos pacientes con soplo cardiaco aumenta su efectividad (15).

El tratamiento inicial a todos los pacientes fue el uso de antibióticos, en el caso de desarrollo o empeoramiento de la insuficiencia cardiaca el paciente debe ser evaluado inmediatamente para cirugía cardiaca, igual al manejo reportado en otros casos (16). En esta investigación sólo $23 \%$ requirieron manejo quirúrgico, entre ellos tres pacientes que finalmente requirieron inserción de marcapasos por la gravedad de la patología (bloqueo AV de alto grado). Las principales complicaciones son a nivel cardiaco y neurológico siendo la insuficiencia cardiaca su eje principal, de igual forma que procesos hemorrágicos y trombóticos (17-19).

La principal limitación del estudio es la muestra poblacional pequeña, sin embargo, esto se debe a la baja prevalencia de esta patología y se podría reproducir ampliando el tamaño de muestra. Por otro lado, la característica retrospectiva 
del estudio, se considera como otra limitación, puesto que facilita el sesgo de la información; sin embargo, se controla mediante la medición objetiva de los datos.

\section{Conclusiones}

En esta serie de casos se encontró características clínicas y microbiológicas similares a lo reportado en otras investigaciones nacionales e internacionales. Afectando con mayor frecuencia a personas del sexo masculino, siendo el principal el Staphylococcus aureus, el agente causal. La fiebre y los signos de vasculitis infecciosa fueron las principales manifestaciones clínicas, afectado con mayor frecuencia la válvula mitral.

\section{Referencias}

1. Jose C, Casabe H. endocarditis infecciosa. Rev Medic. 2008; 68 (2):164-74. Available from: https://www.medicinabuenosaires.com/demo/revistas/vol68-08/2/ v68_n2_p164_174_.pdf

2. Velásquez $\mathbf{O}$. Endocarditis : una patología para actuar con decisión endocarditis : a pathology to act with decision. Rev Col Cardiolog 2013; 1: 13-4.

3. Holland TL, Baddour LM, Bayer AS, Hoen B, Miro JM, Fowler VG. Infective endocarditis HHS Public Access. Nat Rev Dis Prim 2017;2:1-49. Available from: http://www.ncbi.nlm.nih.gov/pubmed/27582414\%0Ahttp://www.pubmedcentral. nih.gov/articlerender.fcgi?artid=PMC5240923\%0Ahttps://www.ncbi.nlm.nih.gov/ pmc/articles/PMC5240923/pdf/nihms815286.pdf

4. Habib G, Lancellotti P, Antunes MJ, Bongiorni MG, Casalta JP, Del Zotti F, et al. Directrices ESC para el tratamiento de la endocarditis infecciosa: el Grupo de trabajo para el tratamiento de la endocarditis infecciosa de la Sociedad Europea de Cardiología (ESC). Eur Heart J. 2015; 36 (44): 3075-128

5. Pant S, Patel NJ, Deshmukh A, Golwala H, Patel N, Badheka A, et al. Tendencias en la incidencia de endocarditis infecciosa, microbiología y reemplazo valvular en los Estados Unidos entre 2000 y 2011. J Am Coll Cardiol. 2015; 65 (19): 2070-6.

6. Bayer AS, Bolger AF, Taubert KA, Wilson W, Steckelberg J, Karchmer AW, et al. Diagnóstico y manejo de la endocarditis infecciosa y sus complicaciones. Circulation. 1998; 98 (25): 2936-48.

7. Damasco PV, Correal JCD, Cruz-Campos AC Da, Wajsbrot BR, Cunha RG da, Fonseca AG da, et al. Epidemiological and clinical profile of infective endocarditis at a Brazilian tertiary care center: an eight-year prospective study. Rev Soc Bras Med Trop. 2019; 52(0): 1-9.

8. Nikolakopoulos A, Koutsogiannis N, Xaplanteri P, Gogos C, Kolonitsiou F, Lekkou A. A Case of Serratia marcescens Endocarditis in a Nonintravenous Drug-Using Male Patient and Review of Literature. Case Rep Infect Dis. 2019; 3 (4): $1-4$.

9. Cheng J, Hu H, Fang W, Shi D, Liang C, Sun Y, et al. Detection of pathogens from resected heart valves of patients with infective endocarditis by next-generation sequencing. Int J Infect Dis 2019; 83: 148-53. Available from: https://doi. org/10.1016/j.ijid.2019.03.007

10. Cahill TJ, Baddour LM, Habib G, Hoen B, Salaun E, Pettersson GB, et al. Challenges in Infective Endocarditis. J Am Coll Cardiol. 2017; 69(3): 325-44.

11. Ambrosioni J, Martinez-Garcia C, Llopis J, Garcia-de-la-Maria C, Hernández-Meneses M, Tellez A, et al. HACEK infective endocarditis: Epidemiology, clinical features, and outcome: A case-control study. Int J Infect Dis. 2018; 76:120-5.

12. Razmi R, Magnusson P. Infektiös endokardit. 2018; 7:1-5.

13. Knebel F, Frumkin D, Flachskampf FA. Infective Endocarditis. Dtsch Medizinische Wochenschrift. 2019; 144(2): 114-27.

14. Agus HZ, Kahraman S, Arslan C, Babur G, Agus HZ, Kahraman S, et al. Characterization, epidemiological profile and risk factors for clinical outcome of infective endocarditis from a tertiary care centre in Turkey Characterization, epidemiological profile and risk factors for clinical outcome of infective endocarditis from a tertiary care centre in Turkey. Infect Dis. 2013 Sep; 13(6): 523-7.

15. Hernando L, Zamorano JL, Pérez de Isla L, Almería C, Rodrigo JL, Aubele AL, Mataix L, Herrera D, Macaya C. [Associated factors to increased sensitivity in the transthoracic echocardiogram for the diagnosis of infective endocarditis]. Arch Cardiol Mex. 2007 Apr-Jun; 77(2): 94-100.

16. Baddour LM, Wilson WR, Bayer AS, Fowler VG, Tleyjeh IM, Rybak MJ, et al. Infective endocarditis in adults: Diagnosis, antimicrobial therapy, and management of complications: A scientific statement for healthcare professionals from the American Heart Association. Vol. 132, Circulation. 2015. 1435-1486 p.

17. Selton-Suty C, Goehringer F, Venner C, Thivilier C, Huttin O, Hoen B. Complications and prognosis of infective endocarditis. Press Medicale 2019; 48(5): 532-8. Available from: https://doi.org/10.1016/j.lpm.2019.04.002

18. Wang P-E, Sia C-H, Ho AF-W, Tan BY-Q. Reply: Infective endocarditis and its neurological complications. QJM An Int J Med 2019; 6 (6):1-2. Available from: https://academic.oup.com/qjmed/advance- rticle/doi/10.1093/qjmed/ hcz170/5527846

19. Alejandro Olaya-Sánchez, Diana Vargas-Vergara, Lorena Montes-Zabala, Yeison Ávila-Cortés, Luis Miguel Cárcamo-Molina. Descripción clínica, microbiológica y ecocardiográfica de la endocarditis infecciosa. Acta Med Colomb 2019; 44. DOI: https://doi.org/10.36104/amc.2019.1223. 